\title{
Imaging of thrombi and assessment of left atrial appendage function: a prospective study comparing transthoracic and transoesophageal echocardiography
}

\author{
H Omran, W Jung, R Rabahieh, P Wirtz, H Becher, S Illien, R Schimpf, B Lüderitz
}

\begin{abstract}
Objective-To compare the value of current transthoracic echocardiographic systems and transoesophageal echocardiography for assessing left atrial appendage function and imaging thrombi. Design-Single blind prospective study. Patients were first investigated by transthoracic echocardiography and thereafter by a second investigator using transoesophageal echocardiography. The feasibility of imaging the left atrial appendage, recording its velocities, and identifying thrombi within the appendage were determined by both methods.
\end{abstract}

Patients-117 consecutive patients with a stroke or transient neurological deficit. Setting-Tertiary cardiac and neurological care centre.

Results-Imaging of the complete appendage was feasible in $75 \%$ of the patients by transthoracic echocardiography and in 95\% by transoesophageal echocardiography. Both methods were concordant for the detection of thrombi in 10 cases. Transoesophageal echocardiography revealed two additional thrombi. In one of these patients, transthoracic echocardiography was not feasible and in the other the thrombus had been missed by transthoracic examination. In patients with adequate transthoracic echogenicity, the specificity and sensitivity of detecting left atrial appendage thrombi were $100 \%$ and $91 \%$, respectively. Recording of left atrial appendage velocities by transthoracic echocardiography was feasible in $69 \%$ of cases. None of the patients with a velocity $>0.3 \mathrm{~m} / \mathrm{s}$ had left atrial appendage thrombi. In the one patient in whom transthoracic echocardiographic evaluation missed a left atrial appendage thrombus, the peak emptying velocity of the left atrial appendage was $0.25 \mathrm{~m} / \mathrm{s}$.

Conclusions-A new generation echocardiographic system allows for the transthoracic detection of left atrial appendage thrombi and accurate determination of left atrial appendage function in most patients with a neurological deficit. (Heart 1999;81:192-198)

Keywords: echocardiography; left atrial appendage thrombi; stroke; thromboembolism
In patients with stroke of embolic origin it is important to search for a cardiogenic source of thromboembolism. A common location of cardiac thrombi is the left atrial appendage. Previous studies have shown that transoesophageal echocardiography has a much higher sensitivity for detecting left atrial appendage thrombi than transthoracic echocardiography. ${ }^{2}$ Transoesophageal echocardiography is claimed to be superior to transthoracic echocardiography because the transducer may be placed within the oesophagus close to the left atrium, thereby allowing an unrestricted view of the left atrial appendage, and transoesophageal transducers have a higher resolution than transthoracic transducers. ${ }^{3}$ As a consequence transoesophageal echocardiography is often performed in patients with embolic stroke to exclude left atrial appendage thrombi. However, transoesphageal echocardiography is uncomfortable for the patient and carries its own risks. ${ }^{4}$

New generation echocardiographic systems may allow higher transthoracic resolution and may have a better Doppler sensitivity. The primary objective of this study was to compare the value of current transthoracic echocardiographic systems with transoesophageal echocardiography for assessing left atrial appendage function and imaging thrombi. A secondary end point was to analyse the relation between the incidence of thrombi in the left atrial appendage and combined clinical and echocardiographic risk factors for thrombus formation.

\section{Methods}

PATIENTS

The study was conducted as a single blind prospective study at a tertiary cardiac and neurological referral centre. It included adult patients with a suspicion of embolic stroke or a transient neurological deficit ( $\leqslant 24$ hours). Written informed consent was obtained from all patients and the study was approved by the Institutional Review Board of the University of Bonn. The presence of clinical risk factors for thrombi formation was assessed. These risk factors included atrial fibrillation and the presence of mitral stenosis or a mitral prosthesis.

\section{ECHOCARDIOGRAPHIC STUDIES}

Patients were first investigated by transthoracic echocardiography and then examined by a different, blinded investigator using transoesophageal echocardiography. All studies were conducted with commercially available
Accepted for publication 9 October 1998 

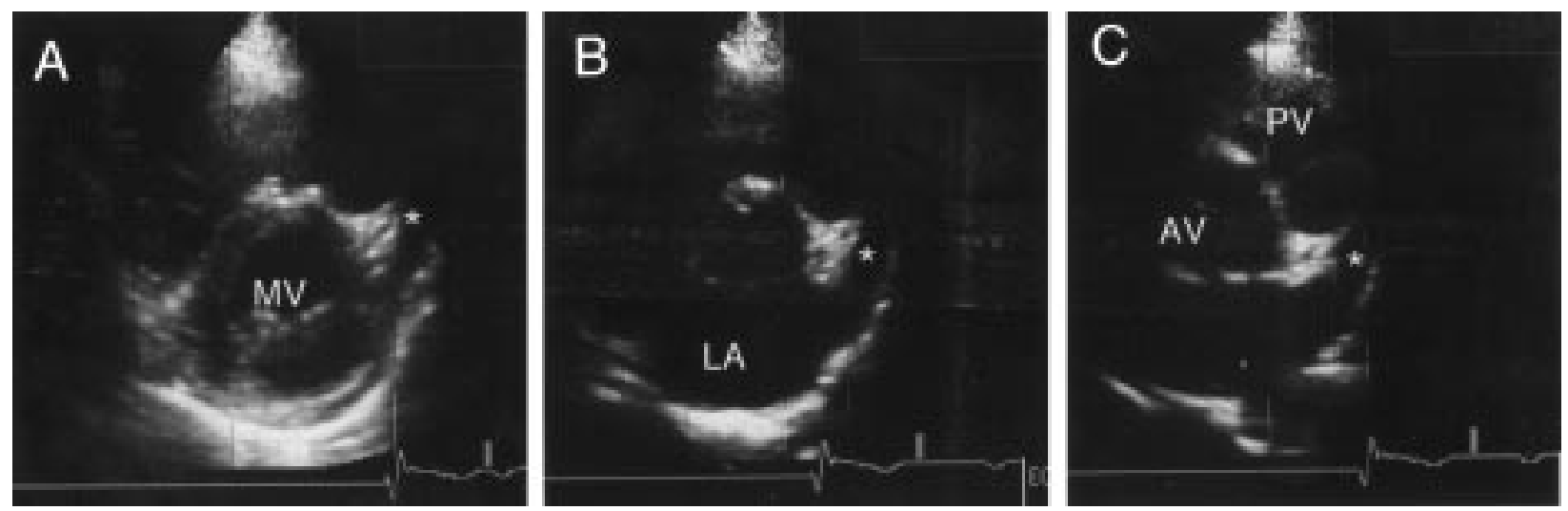

Figure 1 Transthoracic imaging of the left atrial appendage. (A) Standard short axis view of the mitral valve. In this position the left atrial appendage may be partially visualised as indicated by the asterisk. (B) The plane of the beam was then angulated superiorly, so that the aortic valve and the complete body of the left atrial appendage may be seen. (C) The left atrial appendage as seen with the transducer beam angulated laterally towards the pulmonary valve. AV, aortic valve; $L A$, left atrium; $M V$, mitral valve; $P V$, pulmonary valve; ${ }^{\star}$ left atrial appendage.

equipment (System V; Vingmed Sound, Horton, Norway). To allow offline quantitative assessment of the echocardiographic data, examinations were either recorded on videotape or selected cineloops and velocity spectra were digitally transferred to a computer for subsequent analysis.

For transthoracic echocardiography, we used a phased array $3.3 \mathrm{MHz}$ transducer with 128 elements. All patients were examined in the left lateral decubitus position. A single lead electrocardiogram was simultaneously recorded. The left atrium was imaged in the standard, parasternal short and long axis, and apical transducer positions. The left atrial appendage was imaged as described by Herzog et al. ${ }^{5}$ First, we obtained the standard parasternal short axis view at the aortic level; then the plane of the transducer beam was angulated superiorly and laterally until the left atrial appendage was shown (fig 1). To obtain multiple views of the left atrial appendage, we changed the position
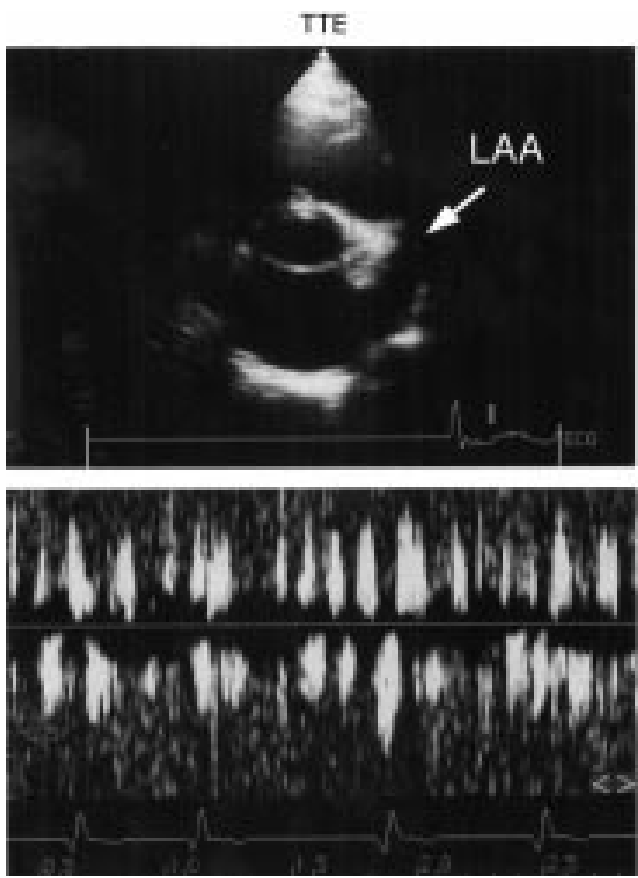

of the transducer to a higher or a lower intercostal space. The imaging plane and gain settings were adjusted to achieve optimum visualisation of the left atrial appendage and the left atrium. The sample volume of the pulsed Doppler was placed $1 \mathrm{~cm}$ into the orifice of the left atrial appendage and the profile of the velocities was recorded (fig 2). We took care to minimise the angle between the Doppler beam and appendage flow. If the angle between the Doppler beam and the flow was $>30^{\circ}$ an angle correction was performed. This was the case in 64 of 81 patients. Cineloops of the left atrium and the left atrial appendage were stored.

Transoesophageal echocardiography was performed with a $5 \mathrm{MHz}$ multiplane transducer. We used topical lignocaine spray and viscous lignocaine solution to anaesthetise the oropharynx before the transoesophageal study. The left atrial appendage was imaged as previously described ${ }^{6}$ : the probe was advanced to
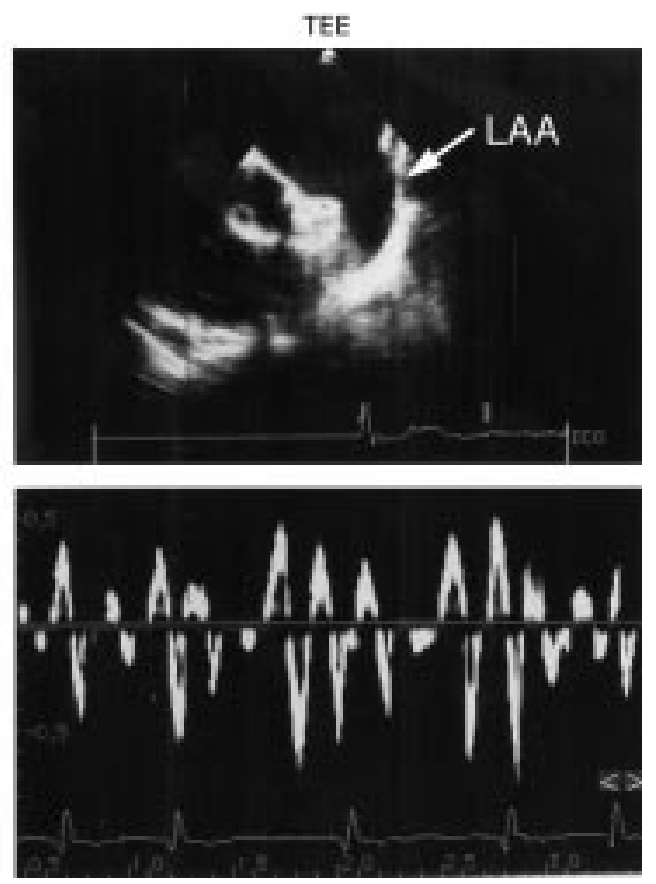

Figure 2 Imaging of the left atrial appendage and recording of the flow velocity profile by transoesophageal (TEE) and transthoracic echocardiography (TTE). LAA, left atrial appendage. 
the mid-oesophagus, posterior to the left atrium; to allow comparison of transthoracic and transoesophageal echocardiographic measurements, the plane of the multiplane probe was adjusted to achieve a short axis view of the aortic valve $\left(45^{\circ}\right)$; to obtain an optimal view of the left atrial appendage, the shaft of the scope was rotated anticlockwise. Multiple views of the left atrial appendage were obtained. Selected cineloops of the left atrium and appendage and velocity spectra of the left atrial appendage were stored for offline analysis.

ECHOCARDIOGRAPHIC DATA ANALYSIS

Echocardiographic evaluations were performed by two additional independent investigators following the original examination. One investigator evaluated the transthoracic, the other the transoesophageal studies. The data were analysed using the evaluation software provided by the manufacturer (Echopac; Vingmed Sound). The feasibility of imaging the left atrial appendage itself, thrombi within the appendage, and spontaneous echo contrast in the left atrium was assessed by both methods. Inadequate transthoracic echogenicity was defined by the following criteria:

- If the transthoracic window did not allow imaging of the left atrial appendage

- If the image of the left atrial appendage was blurred by artefacts

- If the borders of the left atrial appendage could not be separated from the surrounding structures.

A left atrial thrombus was diagnosed by the presence of an echodense mass in the left atrial chamber or appendage with a well defined border to the endocardium. The maximum and minimum area of the left atrial appendage and the peak emptying velocities of the left atrial appendage were measured. Left atrial appendage area was measured by tracing a line starting from the top of the limbus of the left upper pulmonary vein along the appendage's endocardial border. Maximum appendage area was determined during five cardiac cycles and averaged. Peak emptying wavelets were measured during seven consecutive cycles each, and maximum velocities then averaged. We categorised the degree of spontaneous echo contrast on both sides as previously reported, using five grades. ${ }^{7}$ Transthoracic echocardiographic risk factors for thrombus formation in the left atrial chamber or appendage were defined as an enlarged left atrial diameter $(>4$ $\mathrm{cm}$ ) and low peak emptying velocities of the left atrial appendage. A cut off value of $0.3 \mathrm{~m} / \mathrm{s}$ was defined to identify patients with an increased risk of left atrial appendage thrombus formation. ${ }^{89}$

\section{STATISTICAL ANALYSIS}

Descriptive data are presented as the mean (SD). Continuous variables between groups were compared by Student's $t$ test. Nominal data were compared by the $\chi^{2}$ test. A p value 0.05 was considered statistically significant. We assessed the degree of agreement between transthoracic and transoesophageal measure- ments and the interobserver variability for transthoracic measurement of peak emptying velocities of the left atrial appendage as previously described. ${ }^{10}$

\section{Results}

PATIENTS

We enrolled 117 patients (69 male, 48 female; age 54 (14) years) prospectively into the study. Sixty eight patients had suffered a stroke and 49 patients had experienced a transient neurological deficit. Atrial fibrillation was found in 52 patients. Two patients had atrial flutter. The remaining 63 patients were in sinus rhythm. Forty six patients had a history of arterial hypertension, 24 had a known history of coronary heart disease, five had idiopathic dilated cardiomyopathy, and three had mitral stenosis. Seven patients had premature ventricular beats and one had an episode of atrial flutter during transoesophageal examination.

FEASIBILITY OF IMAGING THE LEFT ATRIAL

APPENDAGE, LEFT ATRIAL THROMBI, AND SPONTANEOUS ECHO CONTRAST

Transoesophageal echocardiography was feasible in 116 of 117 patients (99\%). In one patient the transoesophageal probe could not be inserted. The left atrial appendage was completely visualised in 111 of those patients (overall feasibility of imaging the left atrial appendage $95 \%$ ). Transthoracic echocardiographic allowed complete visualisation of the left atrial appendage in 88 of 117 patients $(75 \%)$.

Transthoracic echocardiography detected 10 thrombi in the left atrial appendage (fig 3A and $3 \mathrm{~B})$. One of these patients had a thrombus within the left atrial appendage which extended to the posterior left atrial wall. Seven patients had mobile thrombi, while in the remaining patients they were immobile. All thrombi measured more than $10 \mathrm{~mm}$ in length. The average length and width of all thrombi, as measured by transthoracic echocardiography, was 26 (9) $\mathrm{mm}$ and 14 (7) $\mathrm{mm}$, respectively. Transoesophageal echocardiography confirmed the presence of thrombi and agreed with transthoracic echocardiography in deciding whether thrombi were mobile or immobile in all cases. In two additional patients, thrombi were found in the left atrial appendage during transoesophageal examination. In one of those patients transthoracic echocardiography did not allow complete visualisation of the left atrial appendage owing to inadequate echogenicity, and in the other the thrombus had been missed by transthoracic examination. In the latter case the thrombus was only $4 \mathrm{~mm}$ long and was located in the tip of the appendage. Transoesophageal measurement of the 10 thrombi that were found by transthoracic echocardiography revealed a greater length (28 (7) $\mathrm{mm} ; \mathrm{p}=0.03 ; 95 \%$ confidence interval (CI) -4.4 to $-0.2 \mathrm{~mm}$ ) and width of the thrombi (17 (6) $\mathrm{mm} ; \mathrm{p}=0.003 ; 95 \% \mathrm{CI}-4.0$ to $-1.2 \mathrm{~mm}$ ). If patients with inadequate transthoracic echogenicity are excluded, the specificity and sensitivity of transthoracic 

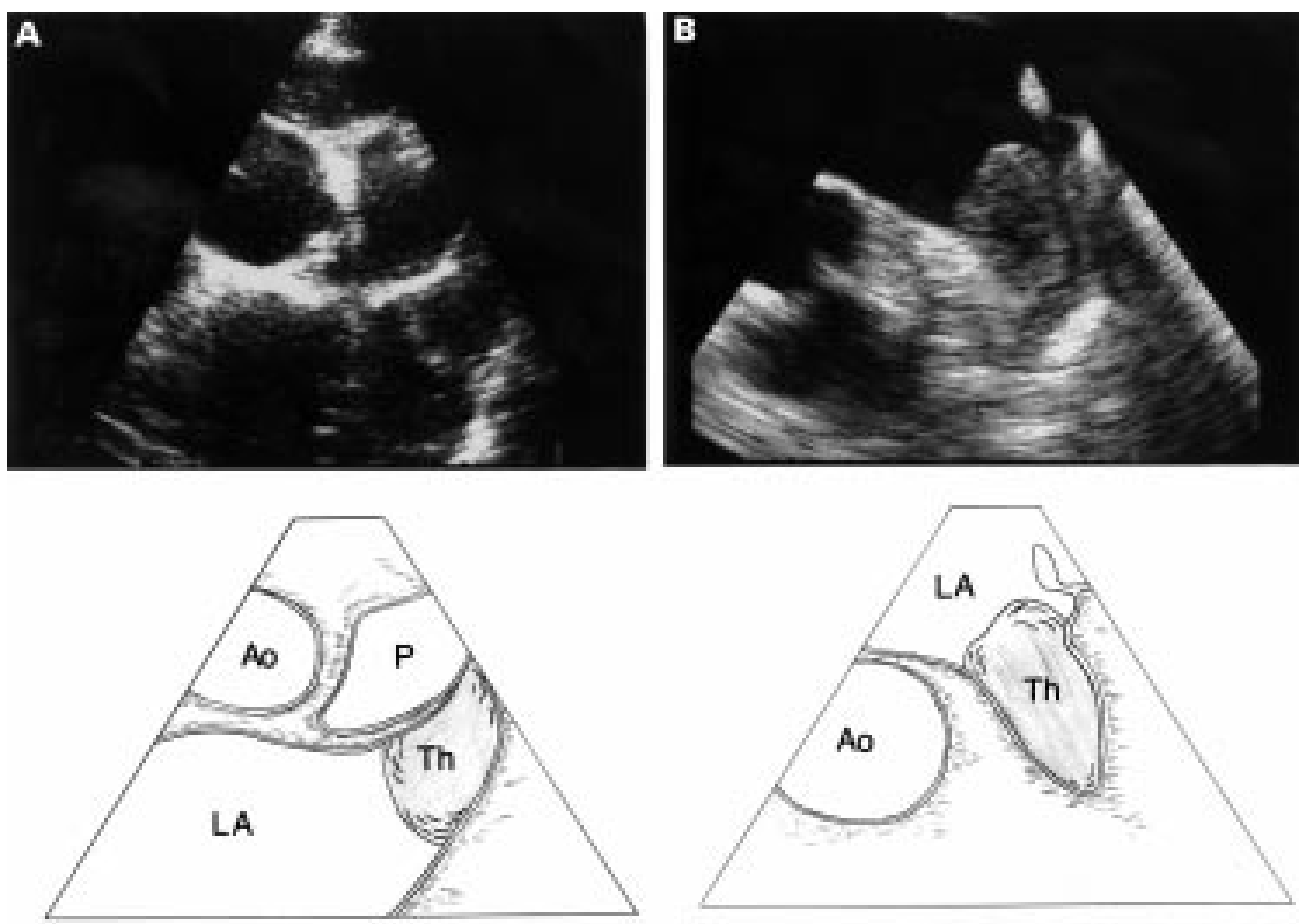

Figure 3 Original images and schematic drawings of a left atrial appendage thrombus as imaged by transthoracic (A) and transoesophageal (B) echocardiography. Ao, aorta; LA, left atrium; P, pulmonary artery; Th, thrombus.

echocardiography for detecting left atrial appendage thrombi were $100 \%$ and $91 \%$, respectively.

Spontaneous echo contrast was found in 55 cases by transoesophageal echocardiography. Sixteen patients had grade $1+, 15$ had grade $2+, 10$ had grade $3+$, and 14 had grade $4+$. In contrast, transthoracic echocardiography detected spontaneous echo contrast in the left atrium only in four cases. All of those patients had spontaneous echo contrast grade $4+$, as classified by transoesophageal echocardiography.

LEFT ATRIAL APPENDAGE SIZE AND PEAK

EMPTYING VELOCITIES

Transthoracic and transoesophageal echocardiographic measurement of the maximum left atrial appendage area did not vary significantly (3.9 (1.4) $\mathrm{cm}^{2}$ v $3.7(1.4) \mathrm{cm}^{2}$ ). In contrast to the measurement of the maximum left atrial appendage area, measurement of the minimum area was feasible in only 100 of 117 patients by means of transoesophageal echocardiography, because contraction of the left atrial appendage caused movement of the appendage outside of the imaging plane. The average minimum left atrial appendage area, as measured by transoesophageal echocardiography, was 2.2 (1.7) $\mathrm{cm}^{2}$. Transthoracic echocardiography allowed imaging of the minimum left atrial appendage area in 63 cases (54\%). Transthoracic measurement of minimal left atrial appendage area was $2.3(2.0) \mathrm{cm}^{2}$.

Measurement of peak emptying velocities was feasible in $97 \%$ of all patients undergoing transoesophageal echocardiography $(n=114$; $0.49(0.22) \mathrm{m} / \mathrm{s})$, whereas this variable was only measurable in $69 \%$ by transthoracic echocardiography $(\mathrm{n}=81 ; 0.50(0.24) \mathrm{m} / \mathrm{s})$. Figure

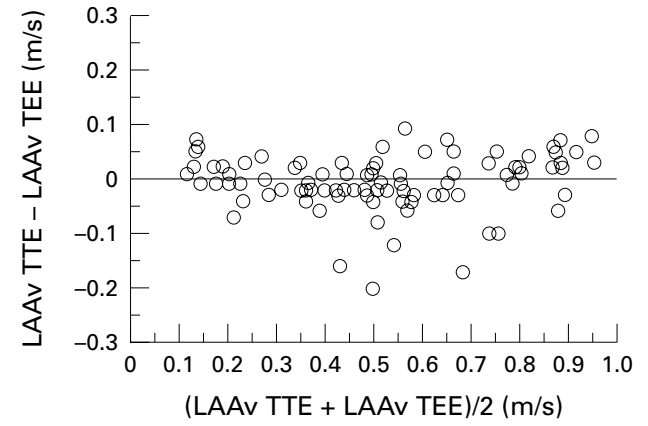

Figure 4 Comparison of transthoracic (TTE) and transoesophageal echocardiography (TEE) for measuring peak emptying velocities of the left atrial appendage (LAAv).

4 shows the correlation of both types of measurement. In patients with thrombi, recording of peak emptying velocities of the left atrial appendage by transthoracic echocardiography was only feasible in three of 10 cases. The individual values of these three patients were $0.21 \mathrm{~m} / \mathrm{s}, 0.25 \mathrm{~m} / \mathrm{s}$, and $0.28 \mathrm{~m} / \mathrm{s}$. In contrast, measurement of peak emptying velocities of the left atrial appendage by transoesophageal echocardiography was feasible in eight of 12 patients with thrombi. The average peak emptying velocity of the left atrial appendage was $0.17(0.06) \mathrm{m} / \mathrm{s}$ in these eight patients. Interobserver variability for the transthoracic measurement of peak emptying velocities of the left atrial appendage was low (fig 5).

RELATION BETWEEN LEFT ATRIAL CHAMBER AND APPENDAGE SIZE AND TRANSTHORACIC VISUALISATION OF LEFT ATRIAL APPENDAGE THROMBI

Left atrial diameter $(5.7(0.7) \mathrm{cm} v 4.1(0.7)$ $\mathrm{cm} ; \mathrm{p}<0.0001 ; 95 \% \mathrm{CI}-2.1$ to $-1.2 \mathrm{~cm})$ and maximum left atrial appendage area $(6.0$ (1.6) 


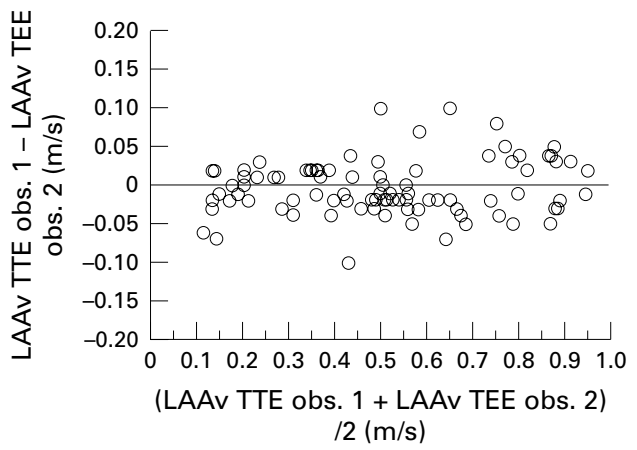

Figure 5 Interobserver variability for measuring peak emptying velocities of the left atrial appendage (LAAv) by transthoracic echocardiography (TTE); obs. 1, observer 1; obs. 2, observer 2.

$\mathrm{cm}^{2}$ v $3.6(1.2) \mathrm{cm}^{2} ; \mathrm{p}<0.0001 ; 95 \% \mathrm{CI}-3.4$ to $-1.4 \mathrm{~cm}^{2}$ ) were significantly larger in patients with thrombi in the appendage, compared with patients without thrombi

RELATION OF THE INCIDENCE OF THROMBI IN THE LEFT ATRIAL APPENDAGE TO COMBINED CLINICAL AND ECHOCARDIOGRAPHIC RISK FACTORS FOR THROMBUS FORMATION Transthoracic echocardiography revealed left atrial appendage thrombi in 10 of 88 patients with adequate transthoracic visualisation of the left atrial appendage. These 10 patients had at least one clinical risk factor for thrombus formation or an enlarged left atrium. Of the remaining 78 patients, only 32 had neither a clinical risk factor nor an enlarged left atrium. In those patients thrombi were not detected by transoesophageal echocardiography. Among the other 46 patients - who had either a clinical risk factor or an enlarged left atrium-peak emptying velocities of the left atrial appendage were $\geqslant 0.3 \mathrm{~m} / \mathrm{s}$ in 30 patient and $<0.3 \mathrm{~m} / \mathrm{s}$ in 16 patients. One patient in the latter group had a thrombus which was detected only by transoesophageal echocardiography. In contrast, none of the 30 patients with a high velocity profile of the left atrial appendage showed a thrombus, despite the presence of a clinical risk factor or an enlarged left atrium.

\section{Discussion}

The primary finding of the present study is that a new generation echocardiographic system allows the transthoracic assessment of left atrial appendage function and detection of left atrial appendage thrombi in $75 \%$ of patients with embolic stroke.

IMAGING OF THE LEFT ATRIAL APPENDAGE

The left atrial appendage is a common site for atrial thrombi and may be the cause of cardiogenic thromboembolism. ${ }^{11}$ Thus in patients with a stroke and a suspicion of embolic origin, it is important to rule out the presence of left atrial appendage thrombi. The results of our study show that the left atrial appendage may be visualised in a large number of patients using of a modern transthoracic echocardiographic system, and that measurements of left atrial appendage areas are comparable with transthoracic and transoesphageal echocardio- graphy. Furthermore, transthoracic echocardiography has a sensitivity of $83 \%$ and a specificity of $100 \%$ for detecting left atrial appendage thrombi. In our study only two of 12 thrombi were not shown by transthoracic echocardiography. In one of these cases, transthoracic echogenicity was not sufficient to visualise the left atrial appendage, while in the other the thrombus was not detected. In the latter case the thrombus was located in the tip of the left atrial appendage and was only $4 \mathrm{~mm}$ long. The failure to image this small thrombus is in accord with previous reports, which showed that thrombi smaller than $10 \mathrm{~mm}$ may not be detected by transthoracic echocardiography. ${ }^{12}{ }^{13}$ Except for one patient, all patients in our study group had left atrial appendage thrombi with a length of $>10 \mathrm{~mm}$, the average length and width being $26 \times 14$ $\mathrm{mm}$. Thus the high sensitivity for detecting thrombi in our study may partly have been the result of the large size of the thrombi.

There are various potential explanations for the high sensitivity and specificity for imaging left atrial appendage thrombi by transthoracic echocardiography in our study. We used a modern digital echocardiographic system with a high dynamic range. Furthermore, a transducer frequency of $3.3 \mathrm{MHz}$ was used, while early studies used frequencies of only 2.25 $\mathrm{MHz}$ and $2.4 \mathrm{MHz}$, respectively. ${ }^{12}{ }^{13}$ The higher transducer frequency used in our study results in a better transthoracic image resolution. In addition, transducer probes used in the earlier studies contained only 32 elements, whereas ours had 128 elements, which increases lateral resolution. The transducer used in our study allowed multiline acquisition, which increases the frame rate. Furthermore, digital image processing and storage significantly improves image quality and offline analysis. Another important factor for enhanced imaging of the left atrial appendage through the transthoracic approach is the significant reduction in transducer probe size compared with the ones used in earlier studies. Thus placement of the transducer in the intercostal space and angulation of the probe is facilitated. Consequently, imaging of the left atrial appendage is simplified and enhanced.

\section{ASSESSMENT OF LEFT ATRIAL APPENDAGE}

FUNCTION

Recent studies employing transoesophageal echocardiography have shown that depressed left atrial appendage function, as assessed by peak emptying velocities of the left atrial appendage and left atrial appendage ejection fraction, is closely correlated with thrombus formation in the appendage and increased thromboembolic risk. ${ }^{8914}$ The results of our study show that transthoracic measurement of peak emptying velocities is feasible in $69 \%$ of patients. Furthermore, our findings demonstrate that transthoracic and transoesophageal measurements of peak emptying velocities of the left atrial appendage correlate closely. The calculation of left atrial appendage ejection fraction requires measurement of the minimum and maximum left atrial appendage area. 
Measurement of the maximum left atrial appendage area was feasible in our study group in $75 \%$ of patients. However, transthoracic imaging of the minimum left atrial appendage was feasible in only $54 \%$ of our patients. This is because of the contraction and rotation of the left atrial appendage during systole, which causes it to move out of the imaging plane. Thus the left atrial appendage ejection fraction could only be calculated in half of the patients in our study group. However, recent studies have shown that appendage velocity measurements are independent of the imaging plane, whereas the ejection fraction of the left atrial appendage is dependent on the plane in which the appendage is viewed. ${ }^{15}$ It therefore seems advantageous to measure peak emptying velocities of the left atrial appendage in order to assess left atrial appendage function.

IMAGING OF SPONTANEOUS ECHO CONTRAST Spontaneous echo contrast accumulation is described as an echogenic swirling pattern of blood flow, which may be detected by transoesophageal echocardiography in conditions favouring stasis of blood in the left atrial chamber and appendage. ${ }^{16}{ }^{17}$ The presence of spontaneous echo contrast has been shown to be associated with a history of thromboembolism and may identify patients with an increased risk of developing stroke or other embolic events. ${ }^{18} 19$ The results of our study show that transthoracic detection of spontaneous echo contrast was feasible in only $9 \%$ of patients in whom it was visualised by transoesophageal echocardiography. Furthermore, transthoracic examination disclosed spontaneous echo contrast only in those patients in whom it was shown to be extremely dense by the transoesophageal method. A possible explanation for the low rate of transthoracic visualisation of spontaneous echo contrast may be that the transthoracic transducer frequency is still significantly lower than the frequency of the transoesophageal probe.

THROMBI IN THE LEFT ATRIAL APPENDAGE AND RISK FACTORS FOR THROMBUS FORMATION The finding of our study that a new generation transthoracic echocardiographic system has a high specificity and sensitivity for detecting left atrial appendage thrombi suggests that additional transoesophageal echocardiographic examination for the confirmation of the presence of a thrombus is not necessary. Nevertheless, our results show that transthoracic echocardiography may miss small thrombi, although the incidence of false negative transthoracic examinations was low $(1 \%)$. It therefore seems useful to preselect patients with a high likelihood of thrombi in order to limit further transoesophageal echocardiographic examination to patients at high risk of thrombus formation. Recently, variables such as the presence of mitral stenosis, mitral prosthesis, atrial fibrillation, and left atrial enlargement have been identified as risk factors for left atrial thrombus formation. ${ }^{20}$ The finding of a left atrial appendage thrombus in patients without these risk factors is unusual. In the latter study only six of
629 patients without clinical risk factors had left atrial appendage thrombi. Owing to the low probability of thrombi in patients without such risk factors, the investigators suggested that transoesophageal echocardiography should be limited to those who have at least one risk factor. Our results support this approach. Patients without any of the risk factors had no thrombi. However, selection of patients on the basis of clinical risk factors and the presence of an enlarged left atrium excluded less than half of our patients $(41 \%)$ from further examination by transoesophageal echocardiography; 46 patients of our study group had at least one clinical risk factor or an enlarged left atrium. Therefore it seems reasonable to look for additional criteria to allow patients with a high probability of thrombi to be selected more precisely.

Recently, low velocities in the left atrial appendage have been correlated with an increased likelihood of left atrial appendage thrombi. ${ }^{8}{ }^{9}$ However, in previous studies it has only been possible to measure this variable by means of transoesophageal echocardiography. Our results show that with improved equipment, velocities may also be recorded by transthoracic echocardiography. In our study, none of the patients with a peak emptying velocity of $>0.3 \mathrm{~m} / \mathrm{s}(\mathrm{n}=30)$, as assessed by transthoracic echocardiography, had a thrombus. Of the remaining 16 patients with at least one clinical risk factor or an enlarged left atrium and a velocity of $<0.3 \mathrm{~m} / \mathrm{s}$, only one had a thrombus $(6 \%)$. In this respect it is important to note that patients in whom thrombi were detected by transthoracic echocardiography either had velocities of $<0.3 \mathrm{~m} / \mathrm{s}(30 \%)$ or velocities that could not be recorded $(70 \%)$. The failure to record velocities in the latter cases may be explained by the fact that they were too low for the Doppler threshold. Thus our results suggest that patients with clinical risk factors may be further risk stratified according to peak emptying velocities of the left atrial appendage. If those velocities are either low or not measurable, transoesophageal echocardiography is recommended.

\section{LIMITATIONS}

With only 117 patients, the sample size of the study is not large enough to provide definite evidence of the role of transthoracic echocardiography for assessing left atrial appendage morphology and function. Our results should be evaluated in further studies. Another limitation of our study is that transthoracic results were only compared with transoesophageal findings. This study design does not take into account the fact that - although it is regarded as the gold standard for imaging left atrial appendage thrombi-transoesophageal echocardiography itself is not absolutely accurate in this respect. Small thrombi between the pectinate muscles or thrombi with an acoustic impedance similar to blood may be overlooked. $^{21} 22$ 
CLINICAL IMPLICATIONS

We compared the accuracy of transthoracic echocardiography for the detection of left atrial appendage thrombi with that of transoesophageal echocardiography, which is considered the current method of choice. If transthoracic echogenicity of patients is optimal, the new generation transthoracic echocardiographic system used is able to detect or exclude left atrial appendage thrombi with a high degree of confidence. Transoesophageal examination may therefore be limited to cases of suboptimal imaging or where there is clinical concern despite negative transthoracic results. Where transthoracic echocardiography is negative with good imaging, and embolic stroke is still suspected, transoesophageal examination is still advised, to rule out other embolic sources such as patent foramen ovale or aortic atheroma.

1 Aschenberg W, Schlüter M, Kremer P, et al. Transesophageal two-dimensional echocardiography for the detection of left atrial appendage thrombus. F $\mathrm{Am}$ Coll Cardiol 1986;7:163-6.

2 Black IW, Hopkins AP, Lee LC, et al. Role of transoesophageal echocardiography in evaluation of cardiogenic embolism. Br Heart f 1991; 66:302-7.

3 Lee RJ, Bartzokis T, Yeoh TK, et al. Enhanced detection of intracardiac sources of cerebral emboli by transesophageal echocardiography. Stroke 1991;22:734-9.

4 Daniel WG, Erbel R, Kasper W, et al. Safety of transesophageal echocardiography. A multicenter survey of 10419 geal echocardiography. A multicenter s
examinations. Circulation 1991;83:817-21.

5 Herzog CA, Bass D, Kane M, et al. Two-dimensional echocardiographic imaging of left atrial appendage echocardiographic imaging of left atrial

6 Seward JB, Khandheria BK, Freeman WK, et al. Multiplane transesophageal echocardiography: image orientation, examination technique, anatomic correlations, and clinical applications. Mayo Clin Proc 1993;68:523-51.

7 Omran H, Jung W, Rabahieh R, et al. Left atrial chamber and appendage function after internal atrial defibrillation: prospective and serial transesophageal echocardiographic study. $\mathcal{F}$ Am Coll Cardiol 1997;29:131-8.
8 Fatkin D, Kelly RP, Feneley MP. Relations between left atrial appendage blood flow velocity, spontaneous echocardiographic contrast and throm
Coll Cardiol 1994;23:961-9.

9 Mügge A, Kühn H, Nikutta P, et al. Assessment of left atrial appendage function by biplane transesophageal echocardiography in patients with nonrheumatic atrial fibrillation: identification of a subgroup of patients at increased embolic risk. F Am Coll Cardiol 1994;23:599-607.

10 Bland JM, Altman DG. Statistical methods for assessing agreement between two methods of clinical measurement. Lancet 1986; i:307-10.

11 Jordan RA, Scheifley CH, Edwards JE. Mural thrombosis and arterial embolism in mitral stenosis. A clinicopathologic study of fifty-one cases. Circulation 1951;3:363-7.

12 Shresta CP, Riley MF, Narciso FV, et al. Two-dimensional Shresta CP, Riley MF, Narciso FV, et al. Two-dimensional
echocardiographic diagnosis of left atrial thrombus in rheumatic heart disease. Circulation 1983;67:341-7.

13 Schweizer P, Bardos P, Erbel R, et al. Detection of left atrial thrombi by echocardiography. Br Heart $\mathcal{F} 1981 ; 45: 148-56$.

14 Pollick C, Taylor D. Assessment of left atrial appendage function by transesophageal echocardiography. Implications for the development of thrombus. Circulation $1991 ; 84: 223-31$.

15 Chan SK, Kannam JP, Douglas PS, et al. Multiplane transesophageal echocardiographic assessment of left atrial appendage anatomy and function. Am $\mathcal{f}$ Cardiol 1995;75: 528-30.

16 Black IW, Hopkins AP, Lee LCL, et al. Left atrial spontaneous echo contrast: a clinical and echocardiographic analysis. $f$ Am Coll Cardio

17 Daniel WG, Nellessen U, Schroder E. Left atrial spontaneous echo contrast in mitral valve disease: an indicator for an increased thromboembolic risk. f Am Coll Cardiol 1988;11: 1204-11

18 Leung DYC, Black IW, Cranney GB, et al. Prognostic implications of left atrial spontaneous echo contrast in nonvalvular atrial fibrillation. f Am Coll Cardiol 1994;24: $755-62$

19 Jones EF, Calafiore P, McNeil JJ, et al. Atrial fibrillation with left atrial spontaneous contrast detected by transesophageal echocardiography is a potent risk factor for stroke. Am f Cardiol 1996;78:425-9.

20 Brickner ME, Friedmann DB, Cigarroa CG, et al. Relation of thrombus in the left atrial appendage by transesophageal echocardiography to clinical risk factors for thrombus formation. Am f Cardiol 1994;74:391-3.

21 Seward JB, Khandheria BK, Oh JK, et al. Critical appraisal of transesophageal echocardiography: limitations, pitfalls, and complications. F Am Soc Echocardiogr 1992;5:288-305.

22 Noma M, Origuchi H, Ueno Y, et al. Sensitivity, specificity, and diagnostic accuracy of transesophageal echocardiographic diagnosis of the left atrial thrombus. Comparison with intraoperative findings [abstract]. Eur Heart $\mathcal{f}$ 1997; 18:1823. 